\title{
Efficacy of statins in improving cognitive functions in vascular dementia
}

\begin{abstract}
Purpose: The purpose of this study was to determine the efficacy and safety of statins in improving cognitive function in patients with vascular dementia. As the most important etiological factors of the disease are atherosclerotic vascular lesions, one of the important areas of treatment is lipid metabolism analysis and drug treatment for dyslipidemia.
\end{abstract}

Methods: 31 patients were selected for the study, ages 65-65 years, 18 males, 13 females. Twenty patients were included in the study group, treated with Simvastatin $(80 \mathrm{mg}$ daily dose). 11 patients were included in the control group. They received placebo therapy. Patients were examined every 4 weeks for 12 weeks using a neuropsychological test with mini-mental scaling, and both groups had low-density lipoprotein and cholesterol levels before and after treatment.

Results: In the 12-week post-treatment group, low-density lipoprotein levels were reduced by $54 \%$ and cholesterol by $48 \%$. Neuropsychological status examination revealed deterioration of cognitive functions and no difference was observed between study and control group data.

Conclusion: Based on the data obtained from our study, it should be noted that correction of lipid metabolism by statins in patients with vascular dementia did not lead to a reduction in cognitive impairment and clinical improvement in patients with vascular dementia.
Volume 10 Issue 6 - 2020

\author{
Tamar lazashvili, Revaz Tabukashvili,Vera \\ Kapetivadze, Zviadi Maglapheridze, Marina \\ Kuparadze, Zurab Grigorashvili \\ Department of Propedeutics of Internal Diseases, State Medical \\ University, Georgia
}

Correspondence: Vera Kapetivadze, Tbilisi, State Medica University, Department of Propedeutics of Internal Diseases, Georgia, Email verakp@rambler.ru

Received: June 16,2020 | Published: November 13, 2020

\section{Introduction}

Dementia is a topical issue in the modern world, including Georgia Statistically, the number of cases is increasing every year, with 2018 as many as 35 million people worldwide suffering from the disease. There were 805 cases of dementia in Georgia in 2014. Since then, these data have been increasing every year, and as of 2017, there have been 1,600 cases of dementia. $70 \%$ of these syndromes are caused by Alzheimer's disease, and $30 \%$ by vascular and other dementia. ${ }^{1}$ More attention has been paid to such a sharp increase in statistical data. According to the WHO experts, a significant problem for older people is CNS disorders, in particular dementia, ${ }^{2-4}$ which is prevalent among individuals aged 75 and above, around 11.2- 17.4\%. Cardiovascular diseases are the second most common cause of dementia. ${ }^{5}$ Vascular dementia encompasses a wide range of disorders and is diverse in both morphological substrates as well as pathophysiological mechanisms and clinical manifestations. The main forms of the disease are multiinfarct dementia, dementia caused by local infarcts of cognitive function zones, multi-infarct, brain hypoperfusion and haemorrhage. ${ }^{6}$ Despite such morphological and pathochemical polymorphisms, the clinical picture of vascular dementia along with cognitive disorders is presented with certain neurological symptoms (paresis, static and coordination disorders, etc.). It is also noteworthy that the cerebral arteriosclerosis is among the most important pathophysiological mechanisms, which develops as a result of micro-atheromatosis and lipohyalinosis of the vascular wall and eventually leads to vascular remodelling, hypoperfusion and white matter damage to the brain. Disease risk factors include arterial hypertension, dyslipidemia, diabetes mellitus, ischemic heart disease, malformations, arrhythmias and more. Depending on the pathogenesis and risk factors, along with etiologic treatment involving the use of acetylcholinesterase inhibitors, neuroprotectors, antidepressants, disease prevention and treatment are one of the important areas of treatment of concomitant diseases, lipid metabolism analysis and dyslipidemia drug correction with HMG CoA reductase inhibitors-statins, bile acid sequences and fibrates. $^{?}$

\section{Materials and methods}

The study was conducted at the Georgian Patriarchate's Therapy Clinic, 31 patients were selected for the study. Inclusion criteria - age 65-65 years, diagnosis, vascular dementia, exclusion criteria: severe somatic abnormalities (heart, kidney, liver failure). Before the study, patients were subjected to physical and neurological examination, mental status was assessed by neuropsychological testing with minimental scaling, lipid metabolism analysis by intravenous blood sample analysis using SIEMENS' Biochemical Complete Automated Analyzer RXL MAX. Patients were assessed 4, 8, and 12 weeks before and at the start of the study. Twenty patients in the study group were given Simvastatin $80 \mathrm{mg}$ daily for three months, 11 patients receiving placebo therapy. Statistical processing of the data was performed using the statistical software Epi-info 7.2.2.6. Version.

\section{Results}

31 patients participated in the study. 18 males (58.1\%), 13 females $(41.9 \%), 20$ patients were included in the study group (64.5\%), in the 11-control group (35.5\%). According to the data obtained before the study, the mean level of low-density lipoproteins in the blood was $9.25 \mathrm{mmol} / \mathrm{l}$ for the study group and $8.9 \mathrm{mmol} / \mathrm{L}$ for the control group. At the end of the study, the mean level in the study group was 5.18 $\mathrm{mmol} / \mathrm{L}$ and in the control group, it was $8.7 \mathrm{mmol} / \mathrm{L}$. The study group showed a decrease in the number of low-density lipoproteins by $48 \%$ and cholesterol by $54 \%$ (Table $1,2 \& 3$ ). 
Table I Determination of low density lipoproteins in blood

\begin{tabular}{|c|c|c|c|c|}
\hline & \multicolumn{2}{|l|}{ Before treatment } & \multicolumn{2}{|l|}{ After treatment } \\
\hline & Study group (20) & Control group (I I) & Study group (20) & Control group (I I) \\
\hline LDL The average concentration in the blood (mmol/L) & 7,34 & 7,41 & 4,96 & 7,38 \\
\hline Cholesterol Level (mmol/L) & 9,25 & 8.92 & 5,18 & 8,76 \\
\hline
\end{tabular}

Table 2 Determination of Cholesterol level

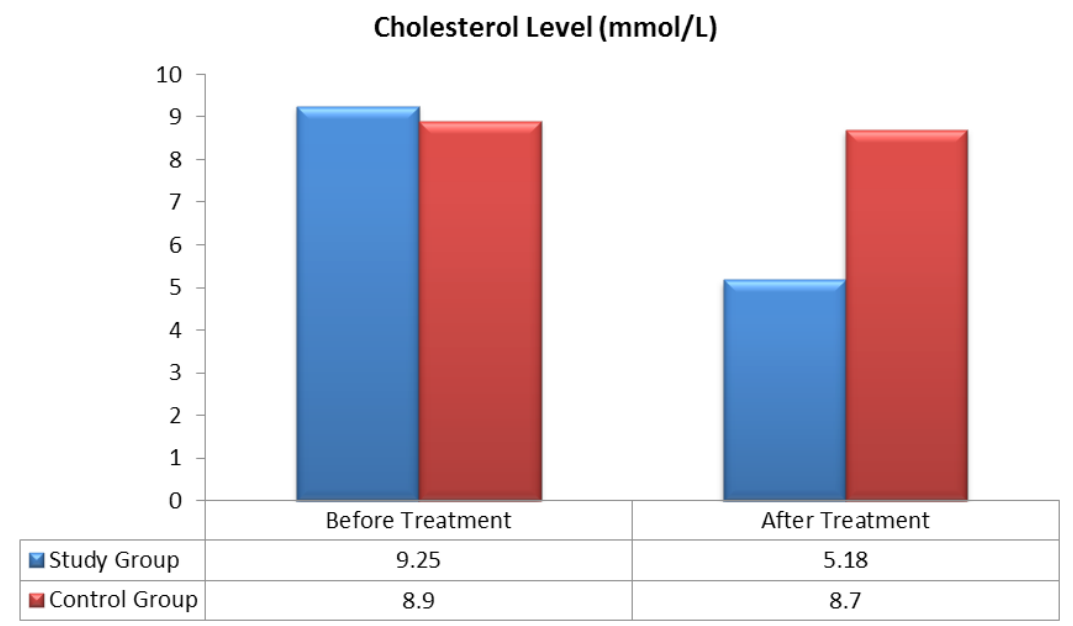

Table 3 Concentration of LDL in blood

\section{LDL The average concentration in the blood ( $\mathrm{mmol} / \mathrm{L})$}

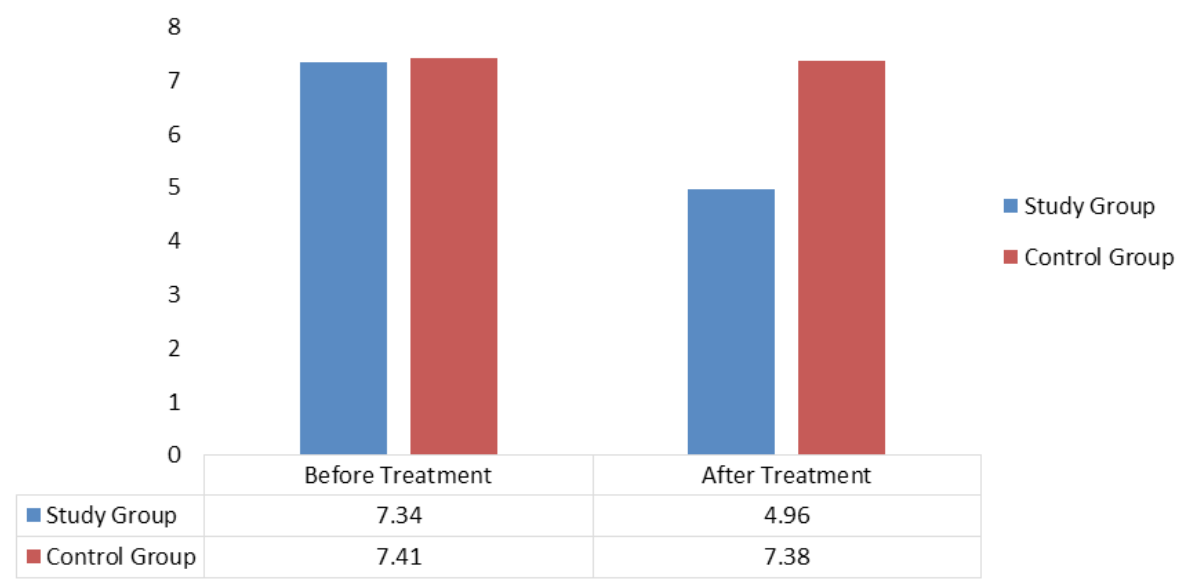

Before the study, the neuropsychological examination of patients assessed memory, orientation, attention, accountability, and constructability through a mini-mental examination scale. The mean score was 16.5 points for the study group and 16,8 points for the control group. A mean decrease in the test score by $3 \%$ in the study group was observed 4 weeks after the start of the study, while the control group remained the same. 8 weeks after, a decrease in the study group was $4.2 \% ; 8.3 \%$ in the control group. 12 weeks after the mean total score decreased by $7.9 \%$ in the study group and $10.7 \%$ in the control group (Table $4 \& 5$ ).

Table 4 Scores of MMSE scale

\begin{tabular}{|c|c|c|}
\hline \multirow{2}{*}{ Test Conduction time } & \multicolumn{2}{|c|}{ Mean arithmetic scores obtained by the MMSE scale } \\
\hline & Study group (20) & Control group (II) \\
\hline Before Treatment & 16,5 & 16,8 \\
\hline 4 weeks after & 16,0 & 16,3 \\
\hline 8 weeks after & 15,8 & 15,4 \\
\hline 12 weeks after & 15,2 & 15,0 \\
\hline
\end{tabular}


Table 5 Average scores of MMSE

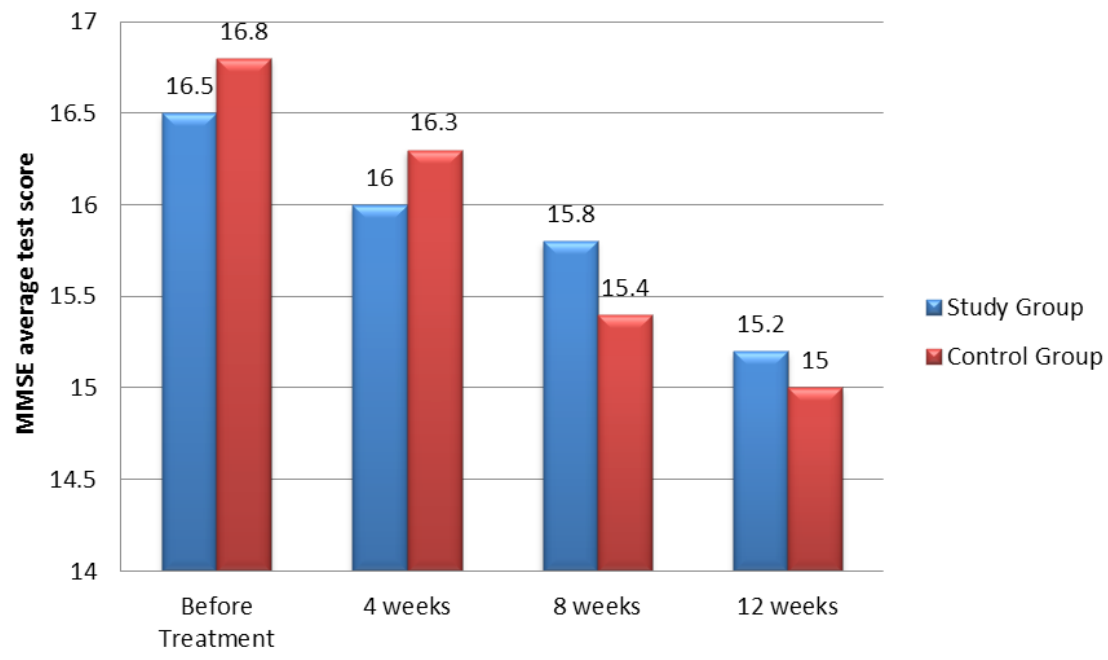

Research has shown that in patients with vascular dementia who have a lipid metabolism disorder, improvement in lipid metabolism following statin treatment does not lead to a decrease in cognitive dysfunction. The study revealed deterioration of cognitive functions in both the study and control groups.

\section{Discussion}

The link between impaired lipid metabolism and impaired cognitive function has not been fully studied, although it is a fact that an increase in low-density lipoprotein and cholesterol concentrations in the blood contributes to atheromatous damage to the blood vessels, including the brain. This results in hypoperfusion and damage to the brain. Depending on the degree of damage, cognitive functions are impaired. The processes are irreversible and eventually lead to severe morbidity and professional disadaptation of patients. ${ }^{8}$ (It may be noted that impairment of cognitive function is a manifestation of the natural process of brain aging, although it may be present to varying degrees physiological changes, moderate or severe cognitive dysfunction and dementia. $^{9}$

Whether lipid metabolism is impaired determines the degree and severity of cognitive dysfunction, is it possible to improve cognitive function in patients with vascular dementia with hypolipidemic therapy? The current data on these issues are inadequate and contradictory, providing no basis for conclusions.

\section{Conclusion}

Based on the data obtained from our study, it should be noted that correction of lipid metabolism by statins in patients with vascular dementia did not lead to a reduction in cognitive impairment and clinical improvement in patients. There was a marked increase in cognitive dysfunction in both the study and control groups. However, it should also be noted that the study was performed on 31 patients in one population of one clinic. Patients with mild to severe cognitive impairments requiring inpatient treatment were selected for the study. It is therefore important to continue research on both inpatient and outpatient patients with mild to moderate cognitive impairment.

\section{Acknowledgments}

None.

\section{Conflicts of interest}

The author declares no conflicts of interest.

\section{References}

1. Gabunia T, Karosanidze I, Kuchava D, et al. Detection and Management of Dementia in General Medical Practice. 2010. p. 4-8.

2. Gelder M, Harrison P, Cowen P. Shorter Oxford Textbook of Psychiatry. 2012.

3. Prince M, Wimo A, Guerchet M. World Alzheimer report 2015: The global impact of dementia. An analysisof prevalence, Alzheimer's Disease International (ADI). 2015.

4. Roman GC, Tatemichi TK, Erkinjunti T, et al. Vascular dementia: diagnostic criteria for research studies. Report of the NINDS-AIREN International Workshop. Neurology. 1993;43:250-260.

5. Solfrizzi V, Panza F, Colaccico AM, et al. Vascular risk factors, incidence of MCI, and rates of progression to dementia. Neurology. 2004;63(10):1882-1891.

6. World Health Organization Neurological Disorders: Public Health Challenges. Switerland: World Health Organization, 2006. p. 204-207.

7. Sulakvelidze M, Melikidze T, Mkhatvari $T$, et al. Management of Hypercholesterolemia in Primary Health Care. West $J$ Med. 1989;150(5):562-568.

8. Larrieu S, Letenneur L, Porgogozo J. Incidence and outcome of mild cognitive impairment in a population based prospective cohort. Neurology. 2002;59(10):1594-1599.

9. Wimo A, Winblad B, Aguero-Torres H, et al. The magnitude of dementia occurrence in the world. Alzheimer Dis Assoc Disord. 2003;17(2):63-67. 